\title{
Carotid artery stenting for symptomatic carotid artery stenosis: An early experience in 15 cases
}

\author{
Ayman A Hassan, ${ }^{a} M D$; Wagih Fawzy, ${ }^{a} M D ;$ Ahmed Abouelnaga, ${ }^{a} M D$; \\ Ahmed K Gabr, ${ }^{a} M D ;$ Ashraf A Essa, ${ }^{b} M D$
}

\begin{abstract}
a) Department of Vascular Surgery, Ain Shams University, Cairo, Egypt. b) Department of Radiology, Bany Sweif University, Bany Sweif, Egypt.
\end{abstract}

\begin{abstract}
Purpose: To demonstrate the outcomes of carotid artery stenting (CAS) in treatment of symptomatic patients with significant extracranial carotid artery stenosis (>70\%) as our early experience in 15 cases.

Methods: Between July 2007 to January 2011, 15 patients for whom successful 15 CAS procedures were done for symptomatic internal carotid artery stenosis more than $70 \%$, were included in the study. All patients underwent pre-procedural assessment in the form of complete history taking, neurological examination, colour duplex ultrasonography (CDU) of carotid arteries, magnetic resonance angiography of carotid arteries and diffusion weighted magnetic resonance imaging (DW-MRI) of the brain. After CAS, patients underwent neurological examination at the day of procedure, DW-MRI of the brain within 72 hours, and clinical examination and CDU of carotid arteries at one month, 6 months and then annually.

Results: Post- procedural death rate was $6.7 \%$. One patient (6.7\%) developed stroke after CAS. New DW-MRI lesions of the brain were found in 5 patients (33.3\%) after CAS, four of them (80\%) remained asymptomatic and were of small volume lesions. Haemorrhagic lesion of the brain was found in one patient (6.7\%) following CAS and was asymptomatic. Stented carotid arteries remained patent in the remaining 14 survived patients with no new neurologic deficits up to one year of follow up following CAS.

Conclusion: Our early experience in using CAS in treatment of symptomatic carotid artery stenosis is promising. CAS seems to be effective and relatively safe therapeutic option in the short and intermediate terms. However, long term risks remain to be determined. As with other new procedures, it is hoped that with increasing experience, we will be able to better identify patients likely to benefit from the procedure, become more comfortable at catheter manipulation and thereby reduce incidence of technical failures and complications of the procedure.

Key words: Carotid artery stenosis, carotid artery stenting.
\end{abstract}

\section{Introduction:}

Stroke is the third leading cause of death in the developed countries after heart diseases and cancer. ${ }^{1}$ A significant percentage of strokes are caused by emboli arising from atherothrombotic carotid artery stenosis which therefore is a risk factor for ischemic stroke. ${ }^{2,3}$

The international trials have clarified the role of carotid endarterectomy (CEA) in the management of selected patients with symptomatic ${ }^{4,5}$ and asymptomatic 6,7 carotid artery disease. Therefore, CEA has rapidly been considered as the standard of care of such patients because of its effectiveness and safety. $2,5,7$

More recently, carotid artery stenting (CAS) which is an endovascular, catheter-based procedure that unblocks narrowing of carotid artery lumen to prevent a stroke, has been proposed as an alternative to CEA for patients at high risk for surgery. Initially, the success of CAS was limited by a rather high rate of neurological events, mainly related to cerebral embolization; ${ }^{8}$ post-procedural neurological 
complication rates as high as $10 \%$ were reported at that time. ${ }^{9}$ Recently, significant improvement of the endovascular techniques emerged and the rate of neurological events has decreased, particularly with the use of protective devices. ${ }^{10}$

The largest clinical trial to date, CREST, ${ }^{11}$ compared CAS to CEA on the collective incidence of any stroke, any heart attack or death. They found that there were no significant differences out to four years of follow up between CAS and CEA when counting all three, but CEA has a higher risk of heart attacks and CAS has a higher risk of minor stroke.

The aim of this study was to demonstrate the outcomes of CAS in treatment of symptomatic patients with significant extracranial carotid artery stenosis $(>70 \%)$ as an our early experience in 15 cases.

\section{Patients and methods:}

\section{Study design:}

This study was performed in two tertiary referral centers in Saudi Arabia during the period between July 2007 and January 2011. We performed prospective non randomized study in order to evaluate the outcomes of CAS in symptomatic patients with extracranial carotid artery disease as in our early experience. Fifteen patients for whom technically successful 15 CAS procedures were done, were enrolled in the study. Two out of 17 attempted cases for CAS $(11.7 \%)$ technically failed due to failure to manipulate the carotid artery lesions and were excluded from the study. Technical failure of these two patients did not affect their pre-intervension neurological status and they were scheduled for CEA. Our indications for treatment were internal carotid artery stenosis of $70 \%$ or more $4,5,9,11$ and the presence of neurological symptoms related to such carotid artery disease. Neurological symptoms in our patients were previous stroke (8 patients) and transient ischemic attacks ( 7 patients). Patients mean age was 66 years (range 50-85 years). All patients were fully informed about the technical aspects and risks of the procedure, and underwent the followings:

\section{Pre-procedural work up:}

- Complete history taking for demographic data, cardiovascular risk factors and neurological symptoms.

- Neurological examination performed by independent neurologist.

- Assessment of the degree of internal carotid artery stenosis and its morphology by colour duplex ultrasonography (CDU) Figure(1A\&1B).

- Cervical and intracranial carotid magnetic resonance angiography (MRA) to verify the diagnosis of carotid artery stenosis and assess intracerebral vasculature.

- Diffusion weighted magnetic resonance imaging (DW-MRI) of the brain performed 24 hours before the procedure.

\section{Post-procedural work up:}

- Neurological examination performed on the day after the procedure.

- DW- MRI of the brain performed within 72 hours after the procedure.

- Clinical and carotid CDU follow up scheduled at one month, 6 months and then annually. The study was approved by the hospitals Ethical Committees and informed consent was obtained from all patients.

\section{Technique of carotid artery stenting (CAS):}

The CAS procedure was performed in the angiography unit under local anaesthesia via percutaneous transfemoral access by a team consisting of vascular surgeons and radiologists. Patient monitoring and management were ensured by an anaesthesiologist. Clopidogrel ( $75 \mathrm{mg}$ ) was given at least 3 days before the procedure and a bolus of intravenous heparin $(100 \mathrm{u} / \mathrm{kg})$ was administered before selective catheterization of common carotid artery. A 7F sheath was placed in the common femoral artery and a $4 \mathrm{~F}$ catheter was introduced for selective cannulation of the common carotid artery. Pre-stenting angiography was performed in the lateral, anteroposterior and oblique planes so as to visualize the severity of the stenosis and the intracerebral vasculature Figure(2A). A long 0.035 inch guidewire (Terumo Stiff exchange) was exchanged for a 7F long sheath which was positioned in the common carotid artery. Under road map guidance, the protective filter (EZ-Boston Scientific) was passed through the internal carotid artery stenosis and deployed at the base of the skull at least $4 \mathrm{~cm}$ beyond the target lesion. The stenosis was then 
dilated with a monorail, 7- or 8-mm diameter, 30-or 40-mm long, self-expanding, metallic stent (Wallstent, Boston Scientific) Figure(2B). After stent deployment, a balloon was inflated to 5 or $6 \mathrm{~mm}$ to minimize residual stenosis. The filter was recaptured. Completion ipsilateral cervical and intracranial carotid angiography was performed to assess technical success and exclude distal cerebral embolization Figure(2C). Finally, after care of groin puncture site was ensured. Antiplatelet medications consisted of clopidogrel (75mg) for 3 months and aspirin (100mg) for life which were given to all patients following the procedure.

\section{Colour duplex ultrasonography (CDU):}

All carotid lesions were detected and imaged by CDU with an ATL 5000 (Philips Medical, Netherlands), using high-frequency probes (4$7 \mathrm{MHz}$ or $5-12 \mathrm{MHz}$ ). The degree of internal carotid artery stenosis and plaque morphology (whether ulcerated or not and degree of calcification) were detected before CAS procedure together with imaging of contralateral carotid and vertebral arteries. Patients were followed up by CDU following CAS procedure to detect restenosis of ipsilateral carotid artery and assess the progress of atherothrombotic disease of the contralateral carotid and vertebral arteries.

Magnetic resonance imaging (MRI):

Before CAS procedure, a baseline cerebral MRI was obtained with a MAGNETOM Symphony $1.5 \mathrm{~T}$ magnetic resonance scanner (Siemens AG Healthcare Sector; Erlanger, Germany). The study included MRA of carotid arteries to verify the diagnosis and DW-MRI of the brain for presence of old ischemic lesion. Following CAS procedure, another DW- MRI was performed within 72 hours. The presence of new hyperintensity in the brain was interpreted as a sign of a new ischemic lesion after CAS Figure(3A\&3B).

\section{Statistical analysis:}

Analysis was performed according to intension-to-treat principle. Data were statistically described as mean $( \pm \mathrm{SD})$ or percentages and were compared using student t-test or Chi-square test as appropriate. A pvalue of less than 0.05 was considered significant. Data were collected and tabulated using Microsoft Excel version 7 (Microsoft Cooperation, NY, USA) and analyzed using SPSS for windows ( statistical package for the social science, version II, SPSS, Inc, Chicago, IL, USA).
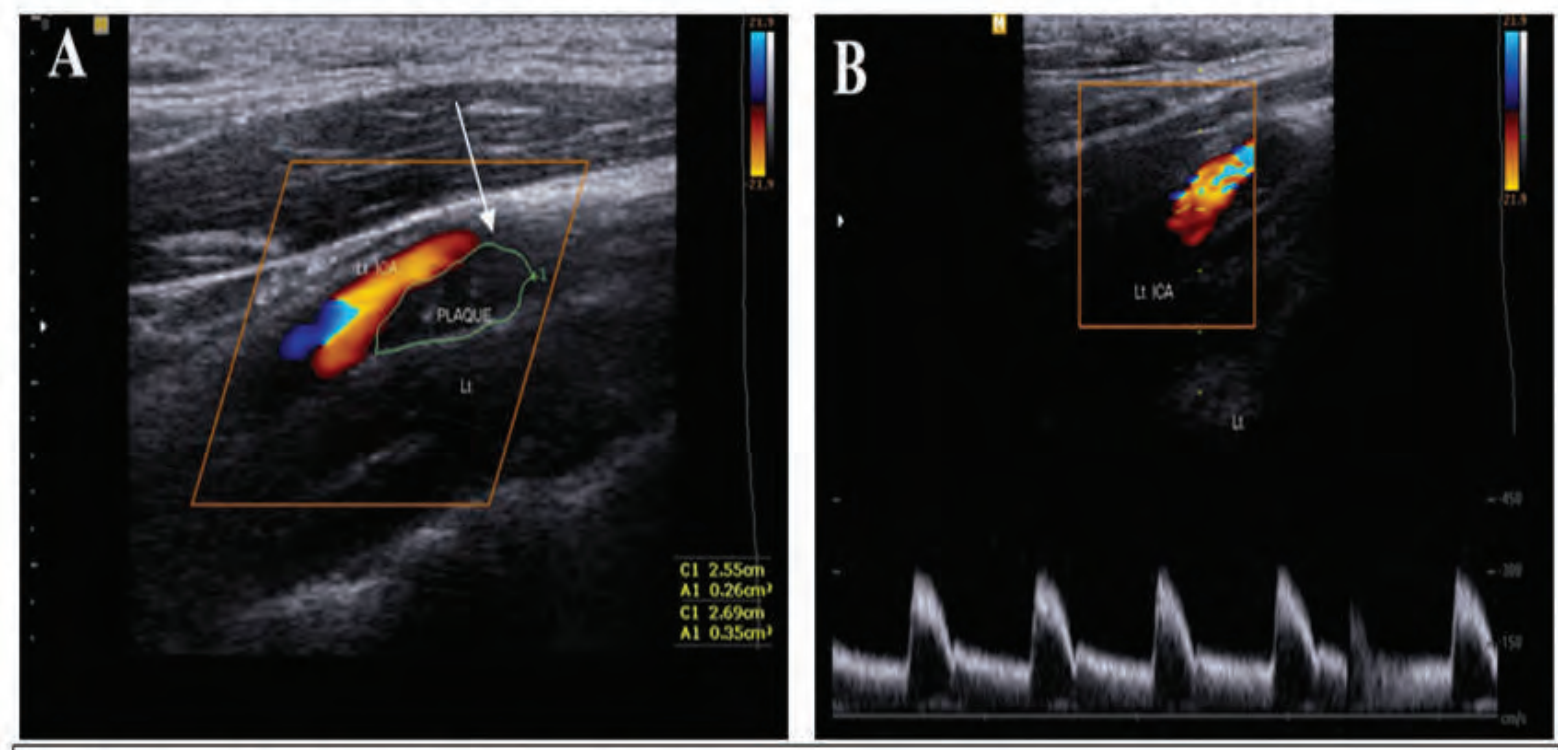

Fig. 1: Colour duplex ultrasound of left carotid arteries showing:

A: Left internal carotid artery $80 \%$ stenosis (arrow) with non-ulcerated plaque.

B: High grade velocity (>300 $\mathrm{ml} /$ second) denoting significant stenosis. 


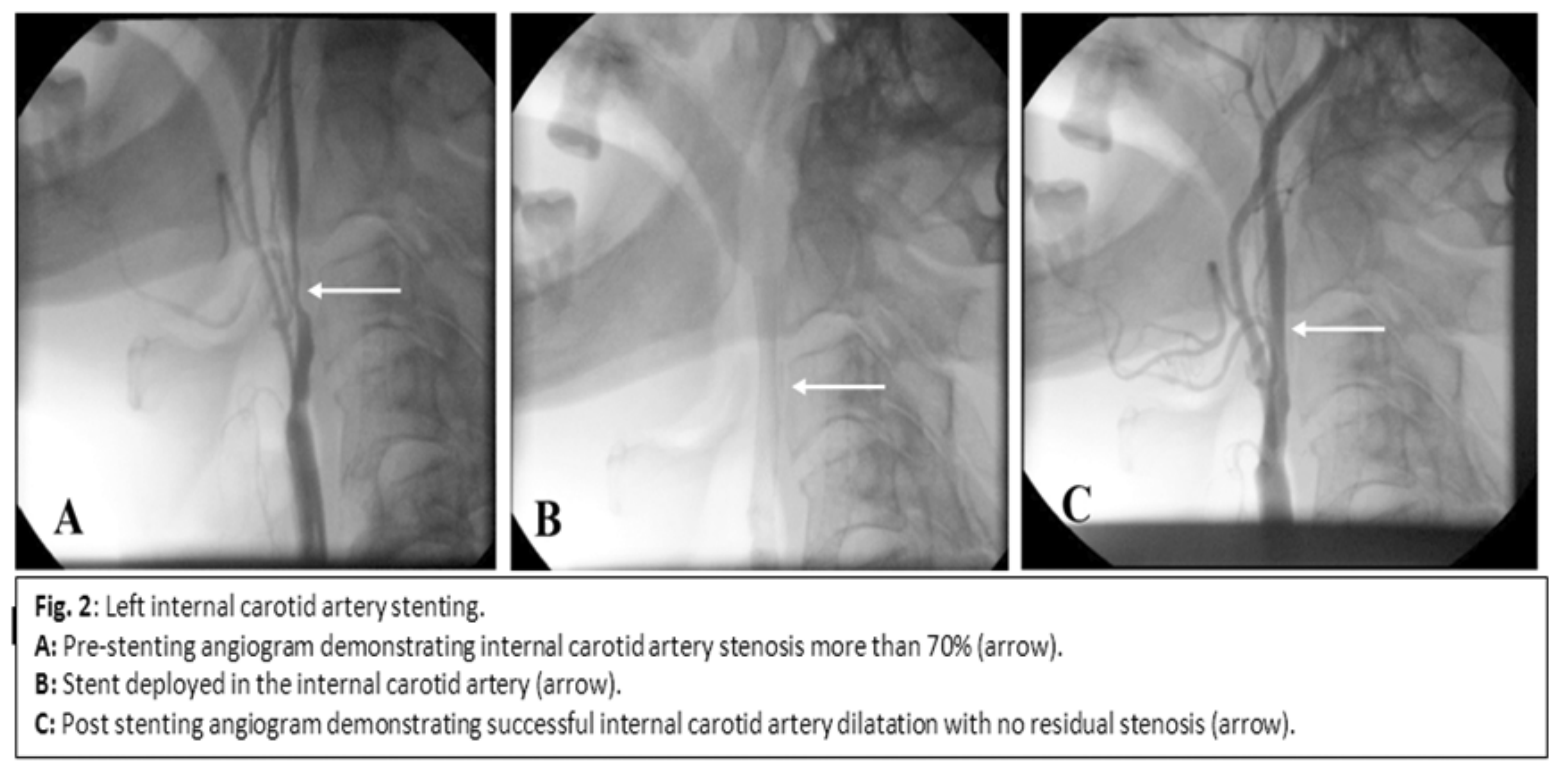

\section{Results:}

The demographic and clinical characteristics of patients are demonstrated in Table(1). They included cardiovascular risk factors with the male gender, hypertension, diabetes mellitus, dyslipidaemia and coronary artery disease being the most common factors.

Table (1): Patients demographic and clinical characteristics.

\begin{tabular}{|l|c|}
\hline Number of patients & 15 \\
\hline Age (year) mean (+SD) & $66(+9.5)$ \\
\hline Gender (male/ female) n.(\%) & $10 / 5(66.7 \%)$ \\
\hline Hypertension n.(\%) & $12(80 \%)$ \\
\hline Diabetes Mellitus n.(\%) & $9(60 \%)$ \\
\hline Dyslipidaemia n.(\%) & $10(66.7 \%)$ \\
\hline Obesity (>10\% of ideal BMI) n.(\%) & $6(40 \%)$ \\
\hline History of smoking n.(\%) & $7(46.7 \%)$ \\
\hline Coronary artery disease n.(\%) & $9(60 \%)$ \\
\hline Peripheral vascular disease n.(\%) & $3(20 \%)$ \\
\hline Chronic obstructive pulmonary disease n.(\%) & $3(20 \%)$ \\
\hline Chronic renal insufficiency n.(\%) & $2(13.3 \%)$ \\
\hline
\end{tabular}

$S D$ : standard deviation; $n$ : number; BMI : body mass index. 
Lesion characteristics of patients at CDU examination before CAS procedure are detailed in Table(2). They included assessment of ipsilateral carotid, contralateral carotid and vertebral arteries.

Table (2): Lesion characteristics at CDU before CAS.

\begin{tabular}{|c|c|}
\hline Number of patients & 15 \\
\hline \multicolumn{2}{|l|}{ Ipsilateral carotid arteries: } \\
\hline \multicolumn{2}{|l|}{ - Stenosis degree } \\
\hline $70-89 \%$ n. $(\%)$ & $10(66.7 \%)$ \\
\hline$\geq 90 \%$ n. $(\%)$ & $5(33.3 \%)$ \\
\hline \multicolumn{2}{|l|}{ - Plaque morphology } \\
\hline Non ulcerated n. (\%) & $12(80 \%)$ \\
\hline Ulcerated n.(\%) & $3(20 \%)$ \\
\hline Non calcified lesion n. (\%) & $6(40 \%)$ \\
\hline Low plaque calcification $(<30 \%)$ n. $(\%)$ & $7(46.7 \%)$ \\
\hline High plaque calcification $(\geq 30 \%)$ n. $(\%)$ & $2(13.3 \%)$ \\
\hline \multicolumn{2}{|l|}{ Contralateral carotid arteries } \\
\hline - No significant stenosis $(<70 \%)$ n. $(\%)$ & $11(73.3 \%)$ \\
\hline - Significant stenosis (70-99\%) n.(\%) & $3(20 \%)$ \\
\hline - Total occlusion n.(\%) & $1(6.7 \%)$ \\
\hline \multicolumn{2}{|l|}{ Vertebral arteries } \\
\hline - Normal flow n.(\%) & $11(73.3 \%)$ \\
\hline - Insufficient flow n.(\%) & $4(26.7 \%)$ \\
\hline
\end{tabular}

$n$ : number ; CDU : colour duplex ultrasonography ; CAS : carotid artery stenting.

Out of 15 patients who underwent CAS procedure, one patient $(6.7 \%)$ died in the first post- procedural day due to myocardial infarction. One patient (6.7\%) developed stroke with clinically evident contralateral hemiplegia. CDU examination of the stented carotid artery of this patient revealed patent vessel while his DW-MRI showed a new large volume hyperintense focus at ipsilateral cerebral hemisphere. This patient improved within 4 weeks of conservative medical treatment. One patient $(6.7 \%)$ got post-procedural groin haematoma which resolved within 3 weeks of conservative treatment. New DW-MRI findings after CAS were found in 5 patients $(33.3 \%)$. All of these findings were in the ipsilateral cerebral hemisphere and related to the anterior cerebral circulation. Four of these findings were of a small lesion volume with no clinical deficits, while only one of them was of a large lesion volume and presented clinically with contralateral hemiplagia as mentioned before. There was no correlation between the new DW-MRI findings and pre-intervension carotid artery stenosis degree or plaque morphology.

One patient (6.7\%) showed small haemorrhagic area in post CAS brain DWMRI related to the anterior cerebral circulation of ipsilateral hemisphere and was of no clinical neurologic deficits. Clinical results and DWMRI brain findings following CAS are summarized in Table(3).

Stented carotid arteries of the remaining 14 survived patients remained patent with no new neurologic deficits up to one year of follow up following CAS. 


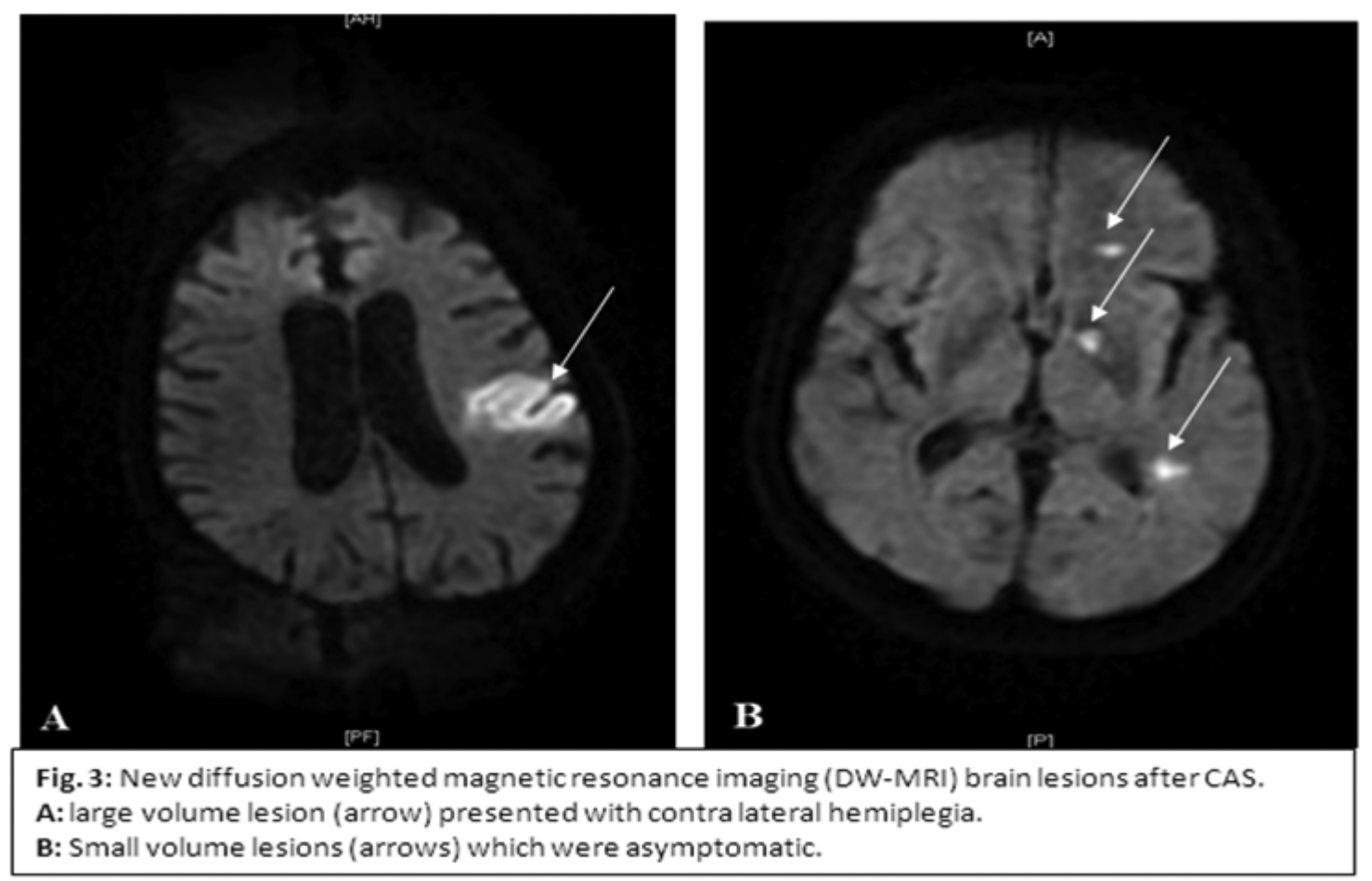

Table (3): Clinical results and DW-MRI brain findings after $C A S$.

\begin{tabular}{|l|c|}
\hline Number of patients & 15 \\
\hline Death n. (\%) & $1(6.7 \%)$ \\
\hline Stroke n. (\%) & $1(6.7 \%)$ \\
\hline Groin haematoma n. (\%) & $1(6.7 \%)$ \\
\hline New DW-MRI findings n.(\%) & $5(33.3 \%)$ \\
\hline Haemorrhagic lesion n. (\%) & $1(6.7 \%)$ \\
\hline
\end{tabular}

\section{Discussion:}

The purpose of treating a stenosis of the carotid artery is to prevent stroke and to otherwise reduce the risk of cerebral ischemia. At present, CEA and CAS are accepted as major therapeutic methods. Nevertheless, CAS has become an attractive treatment for patients because of shorter hospital stays, and the avoidance of general anaesthesia, surgical incision, and the risk of cranial nerve injury. 9,12 The results of CAS have rapidly improved overtime, and recent controlled series show very low post procedural complication rates comparing favorably with CEA. $11,13,14$ However, the superiority of the endovascular approach still needs to be evidenced and several randomized studies are in progress. ${ }^{11,15}$

This study represented our early experience in using CAS as a therapeutic option for carotid artery stenosis. Our data showed death rate of $6.7 \%$ following CAS. The initial results of international carotid stenting study ${ }^{15}$, showed death rate of $4 \%$ which is lower than that of our study, but of no statistical significance. Other studies reported death rates about (2.1 $-5.2 \%$ ) following CAS. ${ }^{13,16,17}$

Our study showed a stroke rate of $6.7 \%$ after CAS. Hakan and coworkers ${ }^{18}$ reported a stroke rate of $5.4 \%$ following CAS which is comparable to our results, while the international carotid stenting study ${ }^{15}$ reported a stroke rate of $4 \%$ which appears to be lower than that of our findings but of no statistical significance. Initially, post CAS neurological complication rates as high as $10 \%$ were reported, ${ }^{9}$ which are significantly higher than that of our results. However, recent controlled series reported stroke rates about (2.1-2.8\%) following CAS, $11,13,16,17$ which are significantly lower than that of our study. This could be explained by the few numbers of cases in our series and that was our early experience. 
New DW-MRI brain lesions were found in $33.3 \%$ of our patients following CAS, $80 \%$ of which was asymptomatic with no additional neurologic deficits. Lacroix and colleagues 19 found new DW-MRI brain lesions of $42.6 \%$ of patients after CAS, $76.9 \%$ of which were asymptomatic, a similar result of our findings. Hakan and coworkers ${ }^{18}$ reported post-CAS new DW-MRI brain lesions of $27 \%$ of patients, a comparable result to our findings. However, only $57 \%$ of these findings were asymptomatic which are lower than that of our results. Furthermore, Loublad and co-author ${ }^{20}$ observed past CAS new DW-MRI brain finding in $21 \%$ of patients, $50 \%$ of which remained asymptomatic. Our data showed that all of the new DW-MRI lesions were located in the ipsilateral cerebral hemisphere and related to anterior cerebral circulation denoting that all emboli was originating from ipsilateral carotid arteries. However, other studies ${ }^{18,19}$ reported that some of these new DW-MRI lesion were located at contralateral cerebral hemisphere or related to posterior cerebral circulation denoting that some of emboli was originating from the aortic arch and stated that even the introduction of a guide-wire or catheter into the aortic arch may be deleterious.

There are several reasons why microemboli cause clinically silent lesions or none at all. The total number of emboli and the size and location of DW-MRI lesions seem to be important in determining whether brain lesions become symptomatic or not. ${ }^{21,22}$ This is true when reviewing our results, we found a DWMRI lesion of large volume symptomatic while those of small volume were asymptomatic, a finding reported by others. ${ }^{18,19}$ No one knows the true clinical significance of cerebral ischemia that is not associated with over neurologic symptoms. Heyer and colleagues ${ }^{23}$ did cognitive testing and found no correlation between DW-MRI findings and neurocognitive dysfunction after CEA. However, long term risks of these silent brain lesions following CAS remain to be determined, a recommendation of other studies. ${ }^{18,19}$ In our study, stented carotid arteries of the survived patients remained patent with no new neurologic deficits up to one year of follow up after CAS. However, we did not test neurocognitive functions for those patients with silent new DW-MRI brain findings following CAS.

Technical failures in our study occurred in $11.7 \%$ of the attempted patients for CAS. These patients were scheduled for CEA and were excluded from final results of the study. Technical failure did not affect the preintervension neurological status of these patients.

We know that we have some limitations in our study. This trial is not controlled and is nonrandomized as we tried to select carotid lesions more feasible for CAS especially in our early cases. Also, there were few numbers of cases in our series, 15 cases in about 3.5 years, which reflects poor referral from neurologists and the belief of people in such community to received medical treatment rather than undergoing such a risky procedure, in their opinion.

\section{Conclusion:}

Our early experience in using CAS in treatment of symptomatic carotid artery stenosis is promising. CAS seems to be effective and relatively safe therapeutic option in the short and intermediate terms. However, long term risks remain to be determined. As with other new procedures, it is hoped that with increasing experience, we will be able to better identify patients likely to benefit from the procedure, become more comfortable at catheter manipulation and thereby reduce incidence of technical failures and complications of the procedure.

\section{References:}

1- Zuromskis T, Wettrholm R, Lindgvist JF, et al: Prevalence of micro-embolic symptomatic high grade carotid artery disease: A transcranial doppler study. Eur J Vasc Endovasc Surg 2008; 35: 534-540.

2- Gasparis AP, Ricotta L, Cuadra SA, et al: High-risk carotid endarterectomy: Fact or fiction. J Vasc Surg 2003; 37: 40-46.

3- Rothwell PM, Wrlow CP: Prediction of benefit from carotid endarterectomy in individual patients: Risk-modeling study. Lancet 1999; 353: 2105-2110.

4- European Carotid Surgery Trialists Collaborative Group: MRC European Carotid Surgery Trial : Interim results for 
symptomatic patients with severe (70-99\%) or with mild (0-29\%) carotid stenosis. Lancet 1991; 337 : 1235-1241.

5- North American Symptomatic Carotid Endarterectomy Trial Collaborators: Beneficial effect of carotid endarterectomy in symptomatic patients with high grade stenosis. N Engl J Med 1991; 325: 445453.

6- Executive Committee for the Asymptomatic Carotid Atherosclerosis Study: Endarterectomy for asymptomatic carotid artery stenosis. JAMA 1995; 273: 14211461.

7- Asymptomatic Carotid Surgery Trial Collaborators: The MRC Asymptomatic Carotid Surgery Trial (ACST): Carotid endarterectomy prevents disabling and fatal carotid territory strokes. Lancet 2004; 363 : 1491-1502.

8- Naylor AR, Bolia A, Abbot RJ, et al: Randomized study of carotid angioplasty and stenting versus carotid endarterectomy: A stopped trial. J Vasc Surg 1998; 28: 326334.

9- CAVATAS Investigators: Endovascular versus Surgical Treatment in Patinets with Carotid Stenosis in the Carotid and Vertebral Artery Transluminal Angioplasty Study (CAVATAS): A Randomized Trial. Lancet 2001; 357; 1729-1937.

10-Parodi J C, La Mura R, Ferreira L M, et al: Initial evaluation of carotid angioplasty and stenting with three different cerebral protection devices. J Vasc Surg 2000; 32: 1127-1136.

11-Brett TG, Hobson RW, Howard G, et al: CREST Investigators: Stenting versus endarterectomy for treatment of carotid artery stenosis. N Engl J Med 2010; 363(1): 11-23.

12-Vadar J S, Wholey M H, Kuntz R E, et al: Protected carotid artery stenting versus endarterectomy in high-risk patients. $N$ Engl J Med 2004; 351 (15): 1493-1501.

13-Carotid Revascularization using endarterectomy or stenting systems (CaRESS) Phase I clinical trial: 1-year results. J Vasc Surg 2005; 42: 213-219.

14-Flach Hz, Ouhlous M, Hendriks J M, et al:
Cerebral ischemia after carotid intervention. J Endovasc Ther 2004; 11: 251-257. 15-International Carotid Stenting Study Investigators: Carotid artery stenting compared with end arterectomy in patients with symptomatic carotid stenosis (International Carotid Stenting Study): An Interim Analysis of a Randomized Controlled Trial. Lancet 2010; 375: 98 997.

16-Hammer F D, Lacroix V, Duprez T, et al: Cerebral micro embolization after protected carotid artery stenting in surgical high-risk patients; results of a 2-year prospective study. J Vasc Surg 2005; 42 (5): 847- 853.

17-Theiss W, Hermanek P, Mathias K, et al: German Societies of Angiology and Radiology. Pre-Cas: A prospective registry of carotid angioplasty and stenting. Stroke 2004; 35: 214-219.

18-Hakan Posacioglu, Cagaray Engin, Celal Cinar, et al: Carotid endarterectomy versus carotid artery stenting; findings in regrad to neuroclinical outcomes and diffusionweighted imaging. Tex Heart Inst J 2008; 35 (4): 395-401.

19-Lacroix V, Hammer F, Astarci P, et al: Ischemic cerebral lesions after carotid surgery and carotid stenting. Eur J Vasc Endovasc Surg 2007; 33: 430-435. 20-Lovblad Ko, Pluschke W, Remonda L, et al: Diffusion-Weighted MRI for monitoring neurovascular intervensions. Neuroradiology 2000; 42 (2): 138-8.

21-Lovblad Ko, Baird AE, Schlang G, et al: Ischemic lesion volumes in acute stroke by diffusion-weighted magnetic resonance imaging correlate with clinical outcome. Ann Neurol 1997; 42 (2): 164-170.

22-Baird AE, Benfield A, Schlang G, et al: Enlargement of human cerebral ischemic lesion volumes measured by diffusionweighted magnetic resonance imaging. Ann Neurol 1997; 41 (5): 581-589.

23-Heyer E J, De La Paz R, Halazun H J, et al: Neuropsychological dysfunction in the absence of structural evidence for cerebral ischemia after uncomplicated carotid endarterectomy. Neurosurgery 2006; 58 (3): 474-480. 